\title{
THE STORAGE RING FOR LOW ENERGY TRITONS AND ITS POSSIBLE APPLICATION
}

\author{
I.Guk, P.Gladkikh, A.Zelinsky, E.Inopin, S.Kononenko, A.Mitsykov, A.Pashenko, Yu.Peresunko, \\ A.Tarasenko, I.Shapoval, A.Shcherbakov \\ NSC KIPT, Kharkov, 61108, Ukraine
}

\section{Abstract}

It is shown that in the circular magnetic system for nuclei of hydrogen isotopes of energies between 20 and $80 \mathrm{KeV} / \mathrm{nucl}$ can provide the damping of both betatron and synchrotron oscillations. A variant of the magnetic lattice of a triton storage ring with a beam energy of 135 $\mathrm{keV}$ is proposed. The main parameters of a stored triton beam (energy spread, amplitude of betatron oscillations, lifetime, etc.) are calculated. It is proposed to use the triton storage ring for generation of neutrons in the reaction $\mathrm{D}(\mathrm{t}, \mathrm{n}){ }^{4} \mathrm{He}$ close to the maximum of the cross section. The integrated intensity and specific power inputs of this source of neutrons is estimated.

\section{INTRODUCTION}

The development of heavy-particle storage rings is connected with the solution of the problem of damping of betatron and synchrotron oscillations. For realizing the damping effect it was proposed in ref. [1] to use the "ionization friction," i.e., to use particle energy losses caused by ionization of the target material located in the chamber of the storage ring. It was also shown that if the particle velocity $v_{p}$ complies with the ratio

$\beta=v_{p} / c<2^{-0.5}$, the ionization energy losses cause a growth in the amplitudes of radial and synchrotron oscillations. Later, the authors of paper [2], developing the idea of [1], demonstrated that, as the sum of all decrements is positive, the instability can be suppressed by coupling the radial motion and the vertical one.

\section{DECREMENTS OF OSCILLATIONS OF HEAVY RELATIVISTIC PARTICLES}

The decrements of betatron and synchrotron oscillations calculated in the refs $[1,2]$ are expressed as:

$$
\begin{aligned}
& \Gamma_{\mathrm{Z}}=\left\langle\frac{c F}{2 \beta \mathrm{E}}\right\rangle \\
& \Gamma_{\mathrm{X}}=\left\langle\frac{c F}{2 \beta E}\left[1-\psi R_{0}\left(K+\frac{\partial \ln F}{\partial x}\right)\right]\right\rangle \\
& \Gamma_{E}=\left\langle\frac{c F}{2 \beta E}\left[\beta^{2} \frac{\partial \ln F}{\partial \ln \gamma}+\psi R_{0}\left(K+\frac{\partial \ln F}{\partial x}\right)\right]\right\rangle
\end{aligned}
$$

where $<>$ denotes averaging over the turn, $F$ are the energy losses per meter, $E$ is the total particle energy, $\gamma$ is the relativistic factor, $\Psi$ is the dispersion function of the magnetic system, $R_{0}$ is reduced radius of the magnetic system, $K$ is the local curvature of closed orbit.

It is evident from expressions (1) - (3) that for providing the damping of all types of oscillations $\left(\Gamma_{X, Z, E}>0\right)$ in the case when the internal target is located in the achromatic section $(\Psi=0)$, the fulfillment of the ratio $\frac{\partial \ln F}{\partial \gamma}>0$ is necessary. The "ionization friction" energy losses dominate over other kinds of losses in the energy range, where this ratio is not satisfied.

As was shown in [3], the only kind of energy losses satisfying the above requirement is inelastic scattering of charged particles by atoms of the internal target.

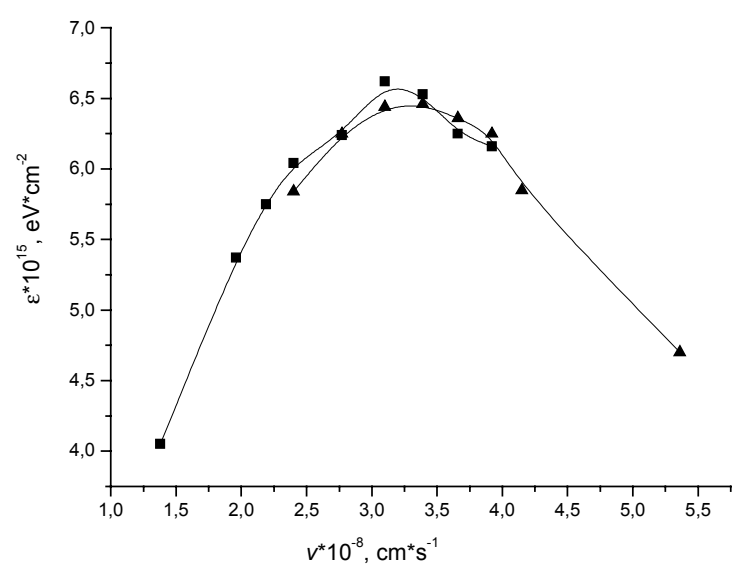

Fig.1 solid squares: data of ref. [4]; solid triangles: data of ref. [5].

The dependence of specific energy losses of hydrogen isotope ions during their passage through atomic hydrogen is shown in Fig. 1. It shoud be noted that the position of extremum in the range $v \sim 3-4^{*} 10^{8} \mathrm{~cm} / \mathrm{s}$ is typical for a wide class of both the scattered particles and the target atoms (see, for example, $[4,5]$ ).

Proceeding from these considerations, the idea of developing of a storage ring of heavy nonrelativistic particles was proposed [3].

The storage of tritons was considered, because the reaction $\mathrm{T}(\mathrm{d}, \mathrm{n})^{4} \mathrm{He}$ has its maximum cross-section at a relative velocity of interacting particles of about $3 * 10^{8}$ $\mathrm{cm} / \mathrm{s}$, and this gives a possibility to realize the damping of 
all types of oscillations. The equilibrium energy of tritons was chosen to be $E_{S} \approx 135 \mathrm{keV}\left(\beta \approx 10^{-2}\right)$.

\section{INTERACTIONS OF A TRITON BEAM WITH THE INTERNAL TARGET}

The main kind of ion beam particle interaction with substance in the range of particle velocities $10^{7}<v<10^{9}$ $\mathrm{cm} / \mathrm{s}$ is the inelastic scattering by the target atoms $[6,7]$.

For calculation of the rms scattering angle of tritons passing through the deuterium target we have used the distribution function given in [8].

To simplify the calculations, it was assumed that $\sin q \approx q$

For $0<p<1$ we have $\overline{\sin ^{2} \vartheta} \cong 0.35 p=5.6 * 10^{-20} \rho_{S}$ ( $\rho_{\mathrm{s}}$ is the surface density of the target), and the decrements of oscillations calculated by formulas (1) - (3) are as follows:

$$
\begin{aligned}
& \Gamma_{\mathrm{X}, \mathrm{Z}} \cong 2.2^{2} * 10^{-15} \rho_{s}\left(\mathrm{~s}^{-1}\right) \\
& \Gamma_{\mathrm{E}} \cong 10^{-15} \rho_{s}\left(\mathrm{~s}^{-1}\right)
\end{aligned}
$$

To calculate the steady-state energy spread in the ion beam, we use the equation for synchrotron oscillations [9]:

$$
d \overline{(\Delta E)^{2}} / d t=\frac{1}{2} \dot{N} \overline{\varepsilon^{2}}-\left(2 \Gamma_{\varepsilon}-\frac{1}{2} \frac{\dot{E}}{E}\right)\left(\overline{\Delta E^{2}}\right),
$$

where $\dot{N} \overline{\varepsilon^{2}}=\frac{d}{d t}\left(\overline{\Delta E_{1}}\right) \varepsilon_{S}, \varepsilon_{S}$ is the average quantum of stopping

$$
d \overline{\Delta \mathrm{E}_{1}} / d t \approx 6.3 * 10^{-15}\left(\mathrm{eV} * \mathrm{~cm}^{2}\right) \rho_{S}\left(v / 2 \pi R_{0}\right)
$$

losses,

For the steady-state case we have:

$$
\overline{(\Delta \mathrm{E})^{2}}=\frac{\Delta \mathrm{E}_{1} \beta c \varepsilon_{S}}{4 \Gamma_{S}}
$$

For a rough estimation of $\overline{(\Delta \mathrm{E})^{2}}$, the average quantum of stopping losses equal to the average energy of atom ionization.

An important problem that appears when devising a storage ring of nonrelativistic hydrogen ions is their neutralization in the interaction with the internal target. The relative amount of neutral particles in the beam after passing a thick target is about $\sigma_{10^{*}}\left(\sigma_{10}+\sigma_{01}\right)^{-1}\left(\sigma_{10}\right.$ and $\sigma_{01}$ are the cross-sections for neutralization and inverse ionization, respectively). The cross sections $\sigma_{10} \approx \sigma_{01}$ are about $10^{-16} \mathrm{~cm}^{2}$ [10] if the velocities of protons are about $3 * 10^{8} \mathrm{~cm} / \mathrm{s}$. For the suppression of the influence of this process on the lifetime of the circulating beam, it would be necessary to use a thin target $\left(\rho_{s}<<10^{16} \mathrm{~cm}^{-2}\right)$, that would cause the increase in the beam damping time.

The neutralization of tritons, in contrast to the charge exchange of protons on hydrogen, is not a resonant process. We now lack the data about the cross section of this process. However, the data presented in [12] show that at the proton energy of about $50 \mathrm{keV}$ the cross section for the reaction $\mathrm{p}+\mathrm{D}_{2} \rightarrow \mathrm{D}+\mathrm{HD}^{+}$is $\sim 10^{-20} \mathrm{~cm}^{2}$, while for the reaction $\mathrm{p}+\mathrm{H}_{2} \rightarrow \mathrm{H}(1 \mathrm{~S})+\mathrm{H}^{+}$it is $10^{-16} \mathrm{~cm}^{2}$. This gives grounds to assume that the cross section for the non-resonant reaction $\mathrm{T}^{+}+\mathrm{D} \rightarrow \mathrm{T}+\mathrm{D}^{+}$is also much less than $10^{-16} \mathrm{~cm}^{2}$. If it appears that this is not true, then the storage ring should be provided with an additional system to secure the neutral particle ionization.

\section{MAGNETIC SYSTEM OF THE TRITON STORAGE RING}

We have performed preliminary calculations of the magnetic system for the storage ring, where experiments with the internal target can be carried out. The maximum physical aperture of the electromagnetic equipment is determined by the ratio:

$$
a_{y \max }(s)=\sqrt{\beta_{t y} * \beta_{y}(s)} \theta_{\max },
$$

where $\beta_{t y}$ is the amplitude function in the y-plane on the azimuth of the target, $\beta_{y}(\mathrm{~s})$ is the current amplitude function along the azimuth of the storage ring, $\vartheta_{\max }$ is the maximum beam divergence accepted by the magnetic system.

In the chosen magnetic system $\beta_{t x} \approx \beta_{t z} \approx 5 \mathrm{~cm}$, $\beta_{t x \max } \approx \beta_{t z \max } \approx 5 \mathrm{~m}$. Thus, the maximum physical aperture $a_{\max }$ is equal to $5 \mathrm{~cm}$ if the maximum acceptance angle $\theta_{\max }$ is equal to $\pm 0.1 \mathrm{rad}$.

The dynamic aperture exceeds the physical one. The simulation of the dynamics of the stored triton beam with due account of its interaction with the internal target is now carried out. The triton beam interaction with the

\begin{tabular}{|c|c|}
\hline Energy losses per turn, keV & $6.1 * 10^{-18} * \rho_{s H}$ \\
\hline $\begin{array}{l}\text { Damping time of betatron } \\
\text { oscillations, sec }\end{array}$ & $6.62 * 10^{17} *<\rho_{s H}>^{-1}$ \\
\hline $\begin{array}{l}\text { Damping time of synchrotron } \\
\text { oscillations, sec }\end{array}$ & $1.44 * 10^{18 *<\rho_{s H}>^{-1}}$ \\
\hline $\begin{array}{l}\text { Beam lifetime due to the } \\
\mathrm{T}(\mathrm{d}, \mathrm{n})^{4} \mathrm{He} \text { reaction, sec }\end{array}$ & $8.3^{*} 10^{14} *<\rho_{s D}>^{-1}$ \\
\hline $\begin{array}{l}\text { Beam lifetime due to single } \\
\text { elastic scattering with the } \\
\text { scattering angle } \theta>\theta_{\max }, \text { sec }\end{array}$ & $10^{15 *} \theta^{2 *} \rho_{s H}{ }^{-1}$ \\
\hline $\begin{array}{l}\text { Amplitude of betatron } \\
\text { oscillations } a_{x, z}, \mathrm{~cm}\end{array}$ & $\sim 0.3$ \\
\hline Steady-state energy spread, keV & 2.1 \\
\hline $\begin{array}{l}\text { Maximum number of stored } \\
\text { particles due to the action of } \\
\text { space charge }\end{array}$ & $\sim 1.6^{*} 10^{11}$ \\
\hline
\end{tabular}
internal target is investigated with the GIANT computer code; beam dynamics in the ring are computed with the MAD code.

Table 1: Parameters of tritium ion beam in the storage ring 


\section{THE TRITON STORAGE RING AS A SOURCE OF NEUTRONS}

It is clear from the data listed in Table 1 that the density of the stopping target is limited by two factors: the capability of the RF system to compensate the energy losses and the capability of the magnetic system to capture the particles after their scattering in the target.

In this connection it seems expedient to separate the functions of stopping and generation of neutrons by using two targets: one, the jet of molecular hydrogen (for stopping); the other, the jet of deuterium plasma (for generation of neutrons). The estimates show that the stopping of tritons in deuterium plasma is less by a factor of $10^{3}$ than in molecular hydrogen.

The expected neutron flux will be about $10^{11}$ neutrons/sec for $\rho_{S D} \sim 5^{*} 10^{18} \mathrm{~cm}^{-2}$ and $\rho_{S H} \sim 0.1 * \rho_{s D}$

The specific power inputs, i.e. the energy input for generation of one neutron, are mainly determined by the $\mathrm{RF}$ power inputs for compensation of inelastic energy losses, and will be about $1.2 * 10^{8} \mathrm{eV} /$ neutron. For comparison, the same parameter for neutron generators or facilities using the $(\gamma, \mathrm{n})$ reaction is about $4 * 10^{9}$ $\mathrm{eV} /$ neutron and $2 * 10^{9} \mathrm{eV} /$ neutron, respectively.

\section{CONCLUSIONS}

Summing up, it is necessary to note the following:

- The use of stopping losses caused by inelastic scattering of particles from atoms of the internal target makes it possible to create storage rings of heavy nonrelativistic particles, because the damping of all types of oscillations is provided in this case;

- The technical realization of such facilities is not too hard; and

- Such facilities can find application in investigations on plasma physics and controlled nuclear fusion, in studies of generation of ultraviolet and soft X-radiation, etc.

\section{REFERENCES}

[1] A.A.Kolomensky, "About Oscillations Decrements in Accelerators with Arbitrary Energy Losses ," Atomnaya Energy, 19, pp. 534-535 (1965) (in Russian).

[2] Yu. M.Ado, V.I. Balbecov. "The Possibility of Ionization Friction Using for Heavy Particles Storing," Atomnaya Energy, 31 (1), pp. 40-44 (1970) (in Russian).

[3] E.V.Inopin, O.S.Tarasenko, "The Tritium Ion Storage Ring as a Neutron Soursce " Ukrainsky Fizichny Journal, 45 (11), pp. 1301-1305 (2000) (in Ukrainian).

[4] J.A.Phillips “ The Energy Loss of Low Energy Proton in Some Gases, ” Phys. Rev., 90 (4), pp. 532-537 (1953).

[5] H.K.Reynolds, D.N.F.Dunbar, W.A.Wenzel, W. Wholing, "The Stopping Cross Section of Gases for Protons of 30-600 keV, ".Phys. Rev., 92 (3), p.p.742748 (1955).

[6] Yu. V.Gott “ The Multiple Scattering of Slow-Speed Particles,” Sov. Phys. Rev., 60, pp.1291-1295 (1971) (in Russian).

[7] H.H.Andersen, J.Bottiger, “ Multiple Scattering of Heavy Ions of keV Energies Transmitted through Thin Carbon Films ," Phys. Rev. B 4, pp. 2105-2110 (1971).

[8] Yu. V.Gott, " Particle Interaction with Matter in Plasma Investigations, " M: "Atomizdat", 1978, 271 p. (in Russian).

[9] G.Bruck, "Cyclic Charged Particle Accelerator,” M.: "'Atomizdat", 1970, 311 p. (in Russian).

[10] R.K.Janev, W.D.Langer, K.Evans, Jr., D.S.Post, "Atomic and Molecular Processes in HydrogenHelium Plasmas" (PPPL-TM-368, June 1985, Plasma Physics Lab., Princeton University). 